\title{
Resolution of dilated cardiomyopathy in an adolescent with change of a failing highly active antiretroviral drug therapy
}

Twalib Olega Aliku' ${ }^{1,2}$, Sulaiman Lubega ${ }^{2}$, Peter Lwabi ${ }^{2}$

1. Department of Paediatrics and Child Health, Gulu University.

2. Uganda Heart Institute, Mulago Hospital Complex.

\section{Abstract}

Background: Cardiovascular dysfunction is a recognized complication of HIV infection in children. Cardiac complications of HIV usually occur late in the course of the disease; they may be associated with drug therapy, and hence become more common as therapy and survival improve. Left ventricular (LV) dysfunction at baseline is a risk factor for death independen of the CD4 cell count, HIV viral load, and neurological disease.

Clinical case: We present the case of a 15 year old girl with HIV who developed left ventricular dysfunction while non-comChe pliant on highly active antictovial the months. Her laboratory evaluaton was significant for elevated ESR an CRP. The ECG showed a sinus tachycardia with diffuse ST-T segment changes and LVH with strain. Initial echo revealed dilated left heart chambers with poor LV systolic function and a small pericardial effusion with the development of an LV thrombus on follow up echo evaluation. She was started on heart failure medicines and had anticoagulation for the LV thrombus. She received adherence counseling and her HAART regimen was changed. Six months after presentation she became asymptomatic with higher $\mathrm{CD} 4$ counts and a normal LV size and function on echo.

Conclusion: Immunological recovery following a switch of a failing or potentially cardiotoxic HAART in addition to improved HAART adherence may result in resolution of left ventricular dysfunction. Early and regular cardiology evaluation may improve outcomes in these patients.

Key words: Dilated Cardiomyopathy, HAART

DOI: http://dx.doi.org/10.4314/ahs.v15i1.39

\section{Background}

Cardiovascular abnormalities in children infected with HIV are frequent and often persistent ${ }^{1}$. Cardiac complications of HIV usually occur late in the course of the disease with prolonged viraemia ${ }^{2-3}$ In HIV infected children not appropriately treated with highly active antiretroviral therapy (HAART), dilated cardiomyopathy is one of the commonest abnormalities seen. These abnormalities may range from subclinical ECG changes to overt heart failure from cardiomyopathy and sudden

\section{Corresponding author}

Twalib Olega Aliku

Department of Paediatrics and Child Health,

Gulu University.

P.O Box 166 Gulu

mobile: +256712551204

Email: aliku90@yahoo.com cardiac death ${ }^{1,4}$. Left ventricular function at baseline has been found to be a risk factor for death that is independent of the CD4 count, HIV viral load and neurological disease $e^{2}$. Long term HAART is cardioprotective for HIV infected children and adolescents ${ }^{5}$, and reduces the risk of cardiomyopathy by $50 \%$ in children compared to those not on HAART

\section{Case report}

We present the case of a 15 year old girl with HIV diagnosed at the age of ten years. For the past five years she received a HAART regimen containing zidovudine, lamivudine, and nevirapine. She was in boarding secondary school and occasionally missed some of her drugs (she had to take at one time ten different drugs). She presented with a two month history of cough, dyspnea, hemoptysis and right hypochondriac pain. She underwent evaluation for pulmonary tuberculosis for her symptoms. Her sputum was negative for alcohol-acid fast bacilli. She was subsequently referred for cardiology consult on the basis of cardiomegaly on the chest radiograph. The physical exam revealed a heart rate of 118 beats per minute, respiratory rate of 24 breaths per minute, $\mathrm{BP}=106 / 79 \mathrm{mmHg}$, and oxygen She had elevated acute phase reactants. The serum saturation of $96 \%$ in room air. She had orthopnea and electrolytes, urea and creatinine were normal At initial mild pedal edema mild pedar edena -10 th percentile) and height $=152 \mathrm{~cm}$ (between 5 th -10 th Percentile). Her cardiovascular exam was remarkable for tachycardia, small volume pulse, displaced apex beat to the 6th intercostal space in the anterior axillary line, a non-sustained apical heave, a third heart sound, basal chest crepitations and a tender hepatomegaly. cardiology evaluation her CD4 count was 51 cells per $\mathrm{ml}$ and the HIV viral load was 36,869 copies per $\mathrm{ml}$ Initial lipid profile was not done. The chest radiography revealed cardiomegaly with cardiothoracic ratio of 0.7 interstitial edema and blunted left costophrenic angle. Her ECG showed a sinus tachycardia with heart rate of 122 beats per minute, diffuse ST-T segment changes and LVH with strain (see figure 1)

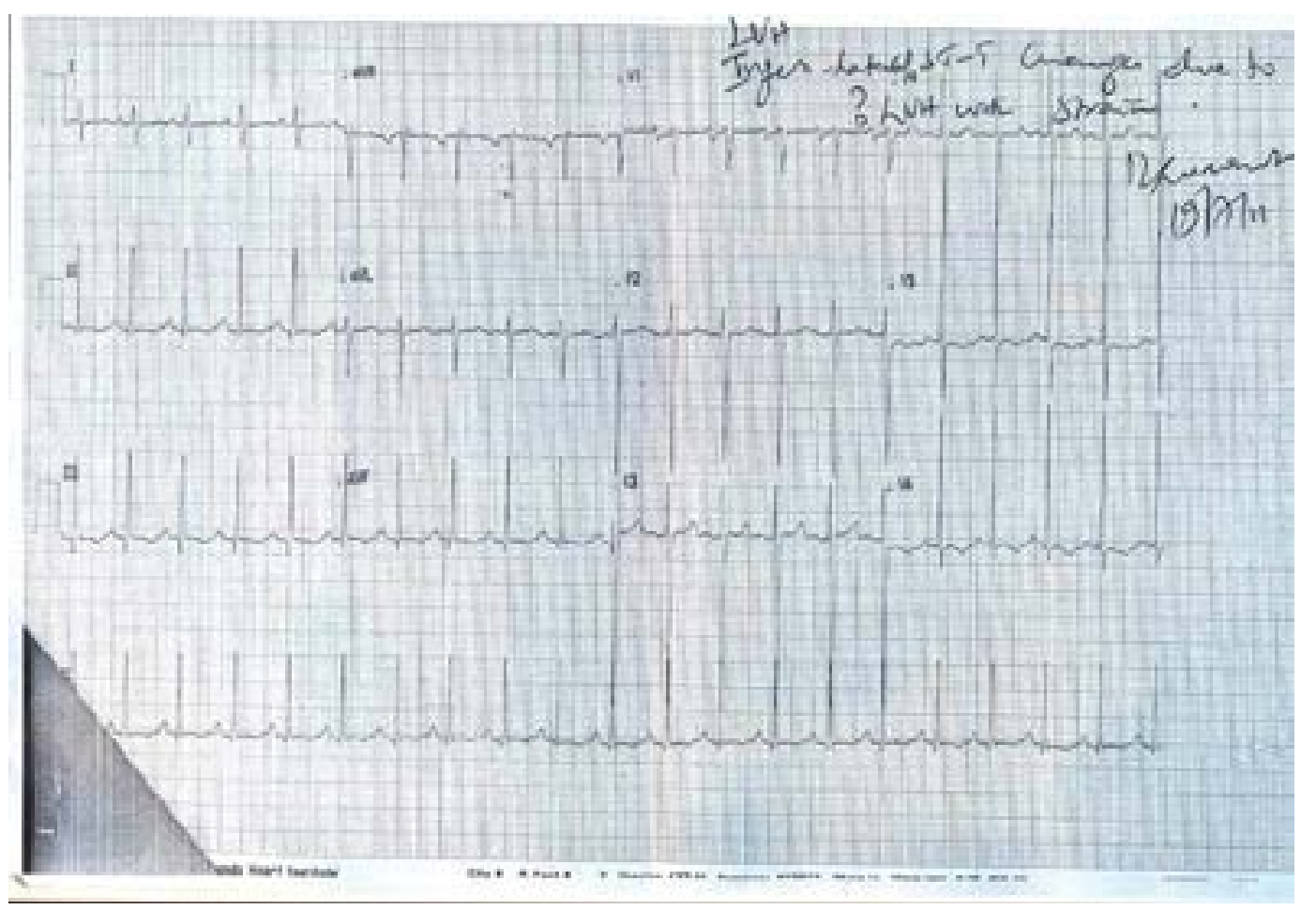

Figure1: The ECG of the patient at initial presentation. Note the diffuse Inferolateral ST-T changes.

Echo revealed dilated left heart chambers with poor side an antibiotic for pneumonia in view of the elevated $\mathrm{LV}$ systolic function ( $\mathrm{LVEDd}=6.2 \mathrm{~cm}, \mathrm{Z}$-score $=3.65$; acute phase reactants. She received counseling on the $\mathrm{LAD}=4.4 \mathrm{~cm}, \quad Z$-score $=4.42 ; \quad \mathrm{FS}=17 \%, \quad \mathrm{EF}=35 \%$ ). importance of adherence to HAART. Her therapy was There were no coronary artery abnormalities, no left then switched to abacavir, lamivudine and lopinavir. ventricular outflow obstruction and no coarctation of Two weeks later her symptoms had markedly improved aorta. There were no intracardiac thrombi. There was a but she was getting transient weakness of her limbs. A small pericardial effusion. repeat echo showed spontaneous echo contrast in the left ventricle with intracardiac thrombi in the $\mathrm{LV}$ apex She was started on heart failure medicines (furosemide, measuring $1.5 \mathrm{~cm}$ by $2.1 \mathrm{~cm}$ and a mild pericardial effuspironolactone, digoxin, carvedilol and lisinopril) along- sion. She was started on enoxaparin and then warfarin. 


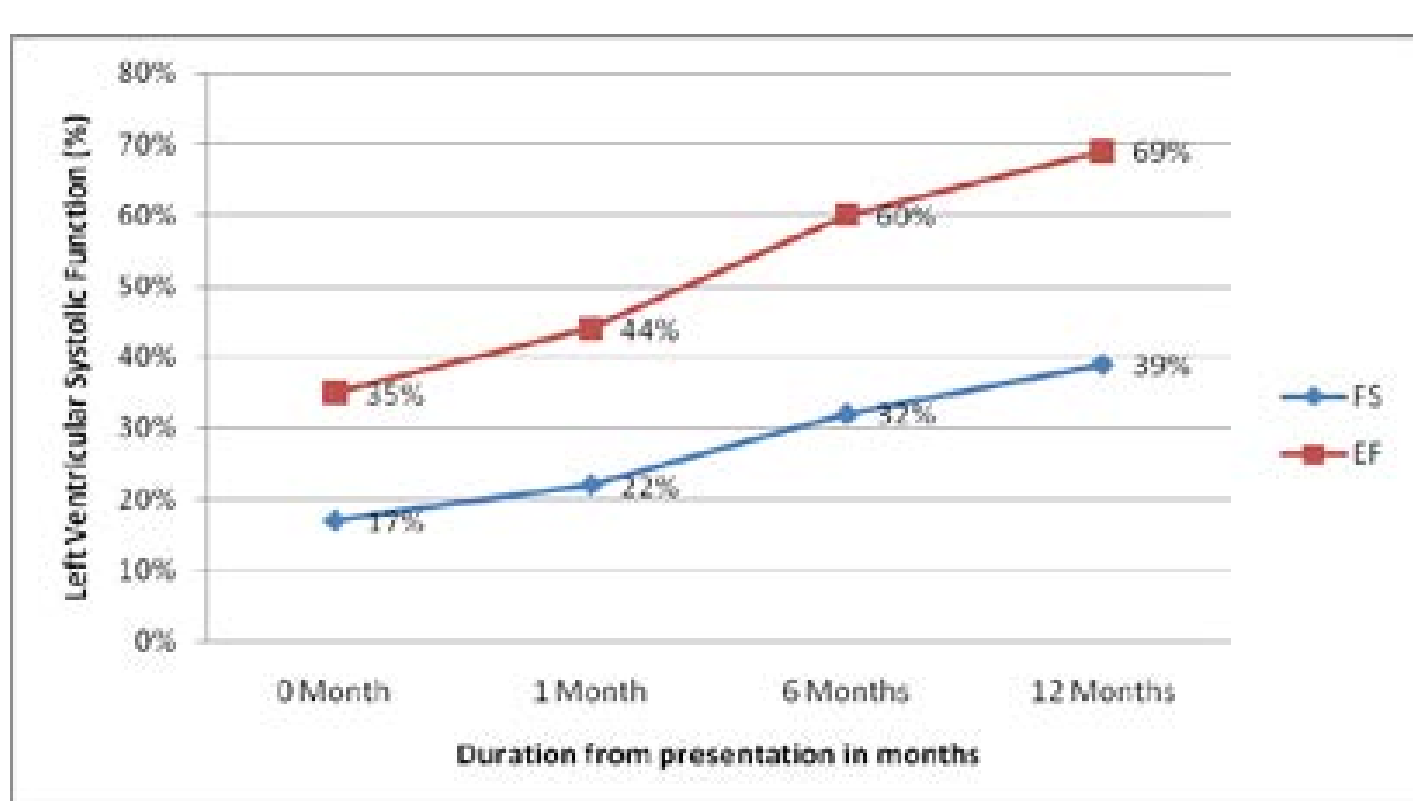

Figure 2: Trends in the patient's left ventricular function. FS, Fractional shortening. EF, Ejection Fraction.

One month later her symptoms had improved. A repeat in her LV function continued to 12 months of follow echo showed no thrombi with an improved LV func- up (see figure 2). Warfarin and the other cardiac meds tion $(\mathrm{FS}=22 \%, \mathrm{EF}=44 \%$ ). Six months after diagnosis, were discontinued except lisinopril. Her CD4 count afshe was asymptomatic. Her echo revealed normal LV ter 6 months from switch of her HAART regimen was size $(\mathrm{LVEDD}=5.1 \mathrm{~cm}, \mathrm{Z}$-score $=1.72)$, with normal sys- 360 copies per $\mathrm{ml}$. The viral load was not done due to tolic function $(\mathrm{FS}=32 \%, \mathrm{EF}=60 \%)$. An upward trend excessive cost. At 12 months of follow up her ECG showed diffuse T-wave inversions (see figure 3 ).

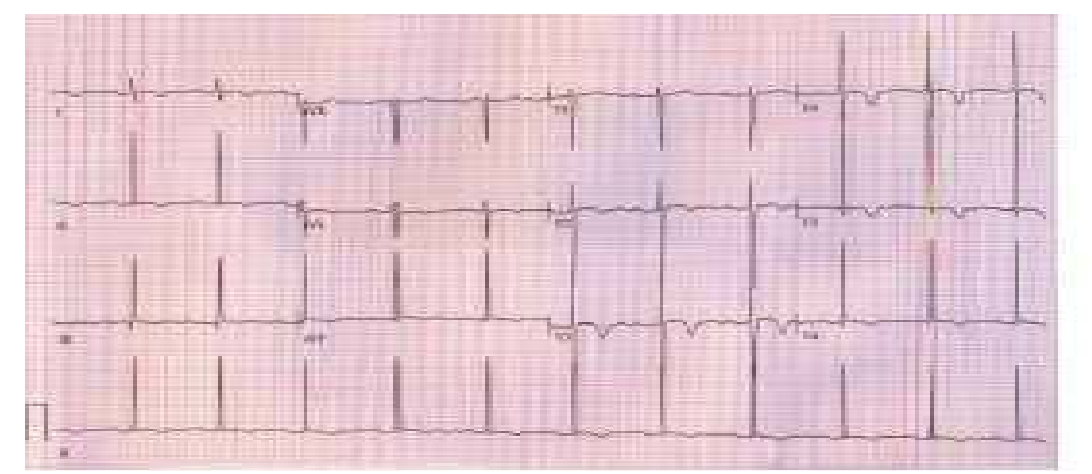

Figure 3: ECG of the patient at 12 months of follow up. Note the persistence of the diffuse T-wave changes in the inferolateral leads.

The fasting lipid profile was abnormal (with raised total ence serious cardiovascular events after the onset of an cholesterol of $5.5 \mathrm{mmol} / 1$, raised LDL of $3.17 \mathrm{mmol} / 1$, AIDS defining illness, especially in those not approprinormal triglycerides of $1.6 \mathrm{mmol} / 1$ and $\mathrm{HDL}$ of ately treated on $\mathrm{HAART}^{7}$. Our patient presented with $1.8 \mathrm{mmol} / \mathrm{l}$ ). She is being followed up every six months. longstanding cough and hemoptysis that prompted initial evaluation for pulmonary tuberculosis. In HIV infected patients, particularly those with advanced dis-

ease; concurrent respiratory infections, pulmonary

\section{Discussion} In HIV infected children less than 10 years of age, $25 \%$ hypertension, anemia, protein energy malnutrition, o may die with chronic cardiac disease and $28 \%$ experi- HIV associated malignancies may alter or obscure the characteristic signs that often define congestive heart failure ${ }^{8}$. In resource limited settings, this could potentially delay the cardiology referral of such patients and contribute to worse outcomes.

The presence of elevated acute phase reactants with tachycardia, diffuse ST-T changes on ECG and severe LV systolic dilated cardiomyopathy in this patient. However, tachycardia is a nonspecific finding in patients with HIV and may be related to autonomic dysfunction. Dilated cardiomyopathy is strongly associated with CD4 counts lower than 100 cells per ml ${ }^{4}$ as was the case in this patient.

Different possible mechanisms may explain the etiology of HIV associated cardiomyopathy. These include the HIV itself, autoimmune response to viral infection, cardiotoxicity or direct mitochondrial injury from therapeutic agents such as zidovudine, nutritional deficiencies and cytotoxic cytokine overexpression'. HIV infected patients with dilated cardiomyopathy are more likely to have myocarditis and a wider spectrum of viral infections than HIV negative patients with idiopathic dilated cardiomyopathy. Immunodeficiency may favor the selection of those viral variants with increased pathogenecity or enhance the cardiovirulence of viral strains ${ }^{9-10}$. Our patient's non adherence to her HAART regimen (in part due to high pill burden sometimes) most likely contributed to the treatment failure.

The combination of low CD4 counts, high viral loads and treatment with potentially cardiotoxic zidovudine in the setting of an infectious insult could have provided the perfect milieu for left ventricular dysfunction in this patient. Change in these risks through switch of HAART regimen to eliminate cardiotoxicity and promote immunological recovery, prompt treatment of concurrent infection and early management of the cardiac dysfunction could have contributed to the better outcome seen in this patient. Tudor et $\mathrm{al}^{11}$ described a similar case of an adolescent with HIV on HAART who had dilated cardiomyopathy. This patient had suboptimal immunological recovery and failure of virologA ssion to undetectable levels. With change in the HAART regiment and immunological recovery, the left ventricular dysfunction improved.

Patients with LV systolic dysfunction are at an increased risk of LV thrombus formation due to associated sta-
However endothelial dysfunction that is common in tients with HIV contributes to a highly procoagulan state $^{12}$. Our patient had features suggestive of a transient ischemic attack and a further echocardiographic examination was able to demonstrate an intracardiac thrombus as the most likely cause.

Even though HAART substantially reduces the risk of cardiomyopathy, patients still remain at increased risk, particularly those with older age at HAART initiation, on zidovudine containing regimen ${ }^{6}$ or those with poor adherence to therapy and treatment failure. The patient is currently being followed regularly since she is on protease inhibitors that are associated with increased cardiovascular risk due to associated dyslipidaemia ${ }^{12}$. Her ECG at 12 months of follow up had subclinical ECG abnormalities.

Echocardiography in children with HIV is recommended in patients at increased cardiovascular risk, those with clinical evidence of cardiovascular disease, unexplained or persistent pulmonary symptoms and every 1 to 2 years thereafter or as clinically indicated ${ }^{7}$. Mondy et al found frequent echocardiographic abnormalities among generally healthy adults on HAART, including LV systolic dysfunction in $18 \%{ }^{13}$. Progressive LV dilatation is common in HIV infected children and may herald the onset of congestive cardiac failure ${ }^{8}$. Rapid onset congestive heart failure has a grim prognosis in HIV infected adults and children, with more than half of patients dying from primary cardiac failure within 12 months of presentation ${ }^{7,14}$

\section{Conclusion}

With standard treatment of the heart failure, improving immunological recovery and optimal virological (suppression from improved adherence) or a change in HAART regimen, patients with HIV may have a resolution of their left ventricular dysfunction. However, they still need a regular cardiology follow up to detect any cardiovascular event, especially in the event of use of HAART with increased cardiovascular toxicity.

\section{References}

1. Lipshultz SE, Easley KA, Orav EJ et al. Left ventricular structure and function in children with the Human immunodeficiency virus: The prospective P2C2HIV multicentre study. Circulation. 1998;97:1246-56.

2. Lipshultz SE, Easley K, Orav EJ et al. Cardiac dys- 
function and mortality in HIV infected children: The prospective P2C2HIV multicentre study. Circulation. 2000;102:542-48.

3. Lubega S, Zirembuzi GW, Lwabi P. Heart disease among children with HIV/AIDS attending the paediatric infectious disease clinic at Mulago Hospital. Afr Health Sciences. 2005;5:219-22.

4. Luginbuhl LM, Orav EJ, McIntosh K, Lipshultz SE. Cardiac morbidity and related mortality in children infected with the Human immunodeficiency virus. JAMA. 1993;269:2869-75.

5. Lipshultz SE, Paige L. W, Wilkinson, Leister EC, et al. Cardiac Status of Children Infected With Human Immunodeficiency Virus Who Are Receiving Long-term Combination Antiretroviral Therapy. Results From the Adolescent Master Protocol of the Multicenter Pediatric HIV/AIDS Cohort Study. Pediatr. 2013;167(6):5207.

6. Patela K, van Dykec RB, Mittlemana MA, Colane $\mathrm{SD}$ et al. The Impact of HAART on Cardiomyopathy among Children and Adolescents Perinatally Infected with HIV-1. for the International Maternal Pediatric Adolescent AIDS Clinical Trials 219/219C Study Team. AIDS. 2013 October; 26(16):2027-37.

7. Al-Attar I, Orav EJ, Exil V et al. Predictors of cardiac morbidity and mortality in children with acquired immunodeficiency syndrome. J Am Coll Cardiol
2003;41:1598. 2003;41:1598.

8. Fisher DS, Lipshultz SE. Cardiovascular abnormalities in HIV infected individuals. In Braunwald's Heart Disease, A text book of cardiovascular medicine, 10th Edition. Editted by Bonow RO, Mann DL, Zipes DP. 2013.

9. Currie PF, Boona NA. Immunopathogenesis of HIV related heart muscle disease: Current perspectives. AIDS. 2003;17:529.

10. Fisher SD, Bowles NE, Towbin JA, Lipshultz SE. Mediators in HIV -associated cardiovascular disease: Focus on cytokines and genes. AIDS. 2003;17:529.

11. Tudor A M, Anca I, Luminos M, Tudor A M, Anca I, Luminos M, Mardarecu M. Dilated cardiomyopathy in a HIV infected adolescent on HAART- a case report. Therapeutics, Pharmacology and Clinical Toxicology. 2010;15(4):302-5.

12. Dubé M P, Lipshultz SE, Fichtenbaum CJ, Greenberg R et al. For Working Group 3. Effects of HIV Infection and Antiretroviral Therapy on the Heart and Vasculature. Circulation. 2008;118:e36-e40.

13. Mondy KE, Gottdiener J, Overton ET, Henry K, Bush TC et al. High prevalence of echocardiographic abnormalities among HIV infected patients in the era of highly active antiretroviral therapy. Clin Infect Dis 2011;52(3):378-86.

14. Ho JE, Hsue PY. Cardiovascular manifestations of HIV infection. Heart 1995;1193,2009. 